\title{
INTEGRAL OPERATORS INDUCED BY THE FOCK KERNEL
}

\author{
MILUTIN DOSTANIĆ AND KEHE ZHU
}

\begin{abstract}
We study the $L^{p}$ boundedness and find the norm of a class of integral operators induced by the reproducing kernel of Fock spaces over $\mathbb{C}^{n}$.
\end{abstract}

\section{INTRODUCTION}

Our analysis will take place in the $n$-dimensional complex Euclidean space $\mathbb{C}^{n}$. For any two points $z=\left(z_{1}, \cdots, z_{n}\right)$ and $w=\left(w_{1}, \cdots, w_{n}\right)$ in $\mathbb{C}^{n}$ we write

$$
\langle z, w\rangle=z_{1} \bar{w}_{1}+\cdots+z_{n} \bar{w}_{n},
$$

and

$$
|z|=\sqrt{\left|z_{1}\right|^{2}+\cdots+\left|z_{n}\right|^{2}} .
$$

For any $t>0$ we consider the Gaussian probability measure

$$
d v_{t}(z)=\left(\frac{t}{\pi}\right)^{n} e^{-t|z|^{2}} d v(z)
$$

on $\mathbb{C}^{n}$, where $d v$ is ordinary Lebesgue volume measure on $\mathbb{C}^{n}$. Let $H\left(\mathbb{C}^{n}\right)$ denote the space of all entire functions on $\mathbb{C}^{n}$. We then define

$$
F_{t}^{p}=L^{p}\left(\mathbb{C}^{n}, d v_{t}\right) \cap H\left(\mathbb{C}^{n}\right)
$$

for $0<p<\infty$. These spaces are often called Fock spaces, or SegalBargman spaces, over $\mathbb{C}^{n}$. See [1][2][3] [7][9][11][16][18].

For $p>0$ and $t>0$ we are going to write

$$
\|f\|_{t, p}=\left[\int_{\mathbb{C}^{n}}|f(z)|^{p} d v_{t}(z)\right]^{\frac{1}{p}},
$$

and

$$
\langle f, g\rangle_{t}=\int_{\mathbb{C}^{n}} f(z) \overline{g(z)} d v_{t}(z) .
$$

Date: December 20, 2006.

2000 Mathematics Subject Classification. 32A36 and 32A15.

Key words and phrases. Fock spaces, Gaussian measure, integral operators.

Dostanić is partialy supported by MNZZS Grant $N^{\circ} O N 144010$.

Zhu is partially supported by the US National Science Foundation. 
It is well known that each Fock space $F_{t}^{p}$ is a closed linear subspace of $L^{p}\left(\mathbb{C}^{n}, d v_{t}\right)$. In particular, in the Hilbert space setting of $L^{2}$, there exists a unique orthogonal projection

$$
P_{t}: L^{2}\left(\mathbb{C}^{n}, d v_{t}\right) \rightarrow F_{t}^{2} .
$$

Furthermore, this projection coincides with the restriction of the following integral operator to $L^{2}\left(\mathbb{C}^{n}, d v_{t}\right)$ :

$$
S_{t} f(z)=\int_{\mathbb{C}^{n}} e^{t\langle z, w\rangle} f(w) d v_{t}(w) .
$$

The integral kernel above,

$$
K_{t}(z, w)=e^{t\langle z, w\rangle}
$$

is the reproducing kernel of $F_{t}^{2}$.

The purpose of this paper is to study the action of the operator $S_{t}$ on the spaces $L^{p}\left(\mathbb{C}^{n}, d v_{s}\right)$, where $s>0$. We also consider the closely related integral operator

$$
T_{t} f(z)=\int_{\mathbb{C}^{n}}\left|K_{t}(z, w)\right| f(w) d v_{t}(w)
$$

or more explicitly,

$$
T_{t} f(z)=\int_{\mathbb{C}^{n}}\left|e^{t\langle z, w\rangle}\right| f(w) d v_{t}(w) .
$$

The main result of the paper is the following.

Main Theorem. Suppose $t>0, s>0$, and $p \geq 1$. Then the following conditions are equivalent.

(a) $T_{t}$ is bounded on $L^{p}\left(\mathbb{C}^{n}, d v_{s}\right)$.

(b) $S_{t}$ is bounded on $L^{p}\left(\mathbb{C}^{n}, d v_{s}\right)$.

(c) $p t=2 s$.

Furthermore, the norms of $T_{t}$ and $S_{t}$ on $L^{p}\left(\mathbb{C}^{n}, d v_{s}\right)$ satisfy

$$
\left\|S_{t}\right\| \leq\left\|T_{t}\right\|=2^{n}
$$

whenever $p t=2 s$.

The equivalence of (a), (b), and (c) is not new; it is implicit in [11] for example. So our main contribution here is the identity $\left\|T_{t}\right\|=2^{n}$. The accurate calculation of the norm of an integral operator is an interesting but often difficult problem. We mention a few successful examples in the literature: the norm of the Cauchy projection on $L^{p}$ of the unit circle is determined in [13], the norm of the Cauchy projection on $L^{p}$ spaces of more general domains is estimated in [5], an asymptotic formula for the norm of 
the Bergman projection on $L^{p}$ spaces of the unit ball is given in [21], and the norm of the Berezin transform on the unit disk is calculated in [6].

As a consequence of the theorem above, we see that the densely defined operator

$$
P_{t}: L^{p}\left(\mathbb{C}^{n}, d v_{t}\right) \rightarrow L^{p}\left(\mathbb{C}^{n}, d v_{t}\right)
$$

is unbounded for any $p \neq 2$. This is in sharp contrast to the theory of Hardy spaces and the theory of Bergman spaces. For example, if $P$ is the Bergman projection for the open unit ball $B_{n}$, that is, if $P$ is the orthogonal projection

$$
P: L^{2}\left(B_{n}, d v\right) \rightarrow L^{2}\left(B_{n}, d v\right) \cap H\left(B_{n}\right),
$$

where $H\left(B_{n}\right)$ is the space of holomorphic functions in $B_{n}$, then

$$
P: L^{p}\left(B_{n}, d v\right) \rightarrow L^{p}\left(B_{n}, d v\right)
$$

is bounded for every $p>1$. A similar result holds for the Cauchy-Szëgo projection in the theory of Hardy spaces. See [14] and [20].

A more general class of integral operators induced by the Bergman kernel on the unit ball $B_{n}$ have been studied in [8][12][19].

We wish to thank Peter Duren and James Tung for bringing to our attention the references [11] and [15].

\section{PRELIMINARIES}

For an $n$-tuple $m=\left(m_{1}, \cdots, m_{n}\right)$ of nonnegative integers we are going to write

$$
|m|=m_{1}+\cdots+m_{n}, \quad m !=m_{1} ! \cdots m_{n} !
$$

If $z \in \mathbb{C}^{n}$, we also write

$$
z^{m}=z_{1}^{m_{1}} \cdots z_{n}^{m_{n}} .
$$

When the dimension $n$ is 1 , we use $d A$ instead of $d v$, and $d A_{t}$ instead of $d v_{t}$. Thus for $t>0$ and $z \in \mathbb{C}$, we have

$$
d A_{t}(z)=\frac{t}{\pi} e^{-t|z|^{2}} d A(z)
$$

where $d A$ is ordinary area measure on the complex plane $\mathbb{C}$.

Lemma 1. Let $m=\left(m_{1}, \cdots, m_{n}\right)$ be an $n$-tuple of nonnegative integers. For any $t>0$ and $p>0$ we have

$$
\int_{\mathbb{C}^{n}}\left|z^{m}\right|^{p} d v_{t}(z)=\prod_{k=1}^{n} \frac{\Gamma\left(\left(p m_{k} / 2\right)+1\right)}{t^{p m_{k} / 2}} .
$$

In particular,

$$
\int_{\mathbb{C}^{n}}\left|z^{m}\right|^{2} d v_{t}(z)=\frac{m !}{t^{|m|}}
$$


Proof. We evaluate the integral in polar coordinates.

$$
\begin{aligned}
\int_{\mathbb{C}^{n}}\left|z^{m}\right|^{p} d v_{t}(z) & =\prod_{k=1}^{n} \int_{\mathbb{C}}\left|z_{k}\right|^{p m_{k}} d A_{t}\left(z_{k}\right) \\
& =\prod_{k=1}^{n} \frac{t}{\pi} \int_{\mathbb{C}}\left|z_{k}\right|^{p m_{k}} e^{-t\left|z_{k}\right|^{2}} d A\left(z_{k}\right) \\
& =\prod_{k=1}^{n} 2 t \int_{0}^{\infty} r^{p m_{k}+1} e^{-t r^{2}} d r \\
& =\prod_{k=1}^{n} t \int_{0}^{\infty} r^{p m_{k} / 2} e^{-t r} d r \\
& =\prod_{k=1}^{n} \frac{1}{t^{p m_{k} / 2}} \int_{0}^{\infty} r^{p m_{k} / 2} e^{-r} d r \\
& =\prod_{k=1}^{n} \frac{\Gamma\left(\left(p m_{k} / 2\right)+1\right)}{t^{p m_{k} / 2}} .
\end{aligned}
$$

The second integral is obviously a special case of the first one.

Recall that the restriction of the operator $S_{t}$ to $L^{2}\left(\mathbb{C}^{n}, d v_{t}\right)$ is nothing but the orthogonal projection onto $F_{t}^{2}$. Consequently, we have the following reproducing formula.

Lemma 2. If $f$ is in $F_{t}^{2}$, then $S_{t} f=f$, that is,

$$
f(a)=\int_{\mathbb{C}^{n}} e^{t\langle a, z\rangle} f(z) d v_{t}(z)
$$

for all $a \in \mathbb{C}^{n}$.

A special case of the reproducing formula above is the following:

$$
K_{t}(a, a)=\int_{\mathbb{C}^{n}}\left|K_{t}(a, z)\right|^{2} d v_{t}(z), \quad a \in \mathbb{C}^{n} .
$$

As an application of this identity, we obtain the following fundamental integrals for powers of kernel functions in Fock spaces.

Lemma 3. Suppose $t>0$ and $s$ is real. Then

$$
\int_{\mathbb{C}^{n}}\left|e^{s\langle z, a\rangle}\right| d v_{t}(z)=e^{s^{2}|a|^{2} / 4 t}
$$

for all $a \in \mathbb{C}^{n}$. 
Proof. It follows from (3) that

$$
\begin{aligned}
\int_{\mathbb{C}^{n}}\left|e^{s\langle z, a\rangle}\right| d v_{t}(z) & =\int_{\mathbb{C}^{n}}\left|e^{t\langle s a / 2 t, z\rangle}\right|^{2} d v_{t}(z) \\
& =\int_{\mathbb{C}^{n}}\left|K_{t}(s a / 2 t, z)\right|^{2} d v_{t}(z) \\
& =K_{t}(s a / 2 t, s a / 2 t) \\
& =e^{s^{2}|a|^{2} / 4 t}
\end{aligned}
$$

This proves the desired identity.

We need two well-known results from the theory of integral operators. The first one concerns the adjoint of a bounded integral operator.

Lemma 4. Suppose $1 \leq p<\infty$ and $1 / p+1 / q=1$. If an integral operator

$$
T f(x)=\int_{X} K(x, y) f(y) d \mu(y)
$$

is bounded on $L^{p}(X, d \mu)$, then its adjoint

$$
T^{*}: L^{q}(X, d \mu) \rightarrow L^{q}(X, d \mu)
$$

is the integral operator given by

$$
T^{*} f(x)=\int_{X} \overline{K(y, x)} f(y) d \mu(y) .
$$

Proof. See [10] for example.

The second result is a useful criterion for the boundedness of integral operators on $L^{p}$ spaces, usually referred to as Schur's test.

Lemma 5. Suppose $H(x, y)$ is a positive kernel and

$$
T f(x)=\int_{X} H(x, y) f(y) d \mu(y)
$$

is the associated integral operator. Let $1<p<\infty$ with $1 / p+1 / q=1$. If there exists a positive function $h(x)$ and positive constants $C_{1}$ and $C_{2}$ such that

$$
\int_{X} H(x, y) h(y)^{q} d \mu(y) \leq C_{1} h(x)^{q}, \quad x \in X,
$$

and

$$
\int_{X} H(x, y) h(x)^{p} d \mu(x) \leq C_{2} h(y)^{p}, \quad y \in X
$$

then the operator $T$ is bounded on $L^{p}(X, d \mu)$. Moreover, the norm of $T$ on $L^{p}(X, d \mu)$ does not exceed $C_{1}^{1 / q} C_{2}^{1 / p}$.

Proof. See [20] for example. 


\section{InTEGRAL OPERATORS INDUCED By THE FOCK KERNEL}

For any $s>0$ we rewrite the integral operators $S_{t}$ and $T_{t}$ defined in (1) and (2) as follows.

$$
S_{t} f(z)=\left(\frac{t}{s}\right)^{n} \int_{\mathbb{C}^{n}} e^{t\langle z, w\rangle+s|w|^{2}-t|w|^{2}} f(w) d v_{s}(w),
$$

and

$$
T_{t} f(z)=\left(\frac{t}{s}\right)^{n} \int_{\mathbb{C}^{n}}\left|e^{t\langle z, w\rangle+s|w|^{2}-t|w|^{2}}\right| f(w) d v_{s}(w) .
$$

It follows from Lemma 4 that the adjoint of $S_{t}$ and $T_{t}$ with respect to the integral pairing

$$
\langle f, g\rangle_{s}=\int_{\mathbb{C}^{n}} f(z) \overline{g(z)} d v_{s}(z)
$$

is given respectively by

$$
S_{t}^{*} f(z)=\left(\frac{t}{s}\right)^{n} e^{(s-t)|z|^{2}} \int_{\mathbb{C}^{n}} e^{t\langle z, w\rangle} f(w) d v_{s}(w),
$$

and

$$
T_{t}^{*} f(z)=\left(\frac{t}{s}\right)^{n} e^{(s-t)|z|^{2}} \int_{\mathbb{C}^{n}}\left|e^{t\langle z, w\rangle}\right| f(w) d v_{s}(w) .
$$

We first prove several necessary conditions for the operator $S_{t}$ to be bounded on $L^{p}\left(\mathbb{C}^{n}, d v_{s}\right)$.

Lemma 6. Suppose $0<p<\infty, t>0$, and $s>0$. If $S_{t}$ is bounded on $L^{p}\left(\mathbb{C}^{n}, d v_{s}\right)$, then $p t \leq 2 s$.

Proof. Consider functions of the following form:

$$
f_{x, k}(z)=e^{-x|z|^{2}} z_{1}^{k}, \quad z \in \mathbb{C}^{n},
$$

where $x>0$ and $k$ is a positive integer.

We first use Lemma 1 to calculate the norm of $f_{x, k}$ in $L^{p}\left(\mathbb{C}^{n}, d v_{s}\right)$.

$$
\begin{aligned}
\int_{\mathbb{C}^{n}}\left|f_{x, k}\right|^{p} d v_{s} & =\left(\frac{s}{\pi}\right)^{n} \int_{\mathbb{C}^{n}}\left|z_{1}\right|^{p k} e^{-(p x+s)|z|^{2}} d v(z) \\
& =\left(\frac{s}{p x+s}\right)^{n} \int_{\mathbb{C}^{n}}\left|z_{1}^{k}\right|^{p} d v_{p x+s}(z) \\
& =\left(\frac{s}{p x+s}\right)^{n} \frac{\Gamma((p k / 2)+1)}{(p x+s)^{p k / 2}}
\end{aligned}
$$


We then calculate the closed form of $S_{t}\left(f_{x, k}\right)$ using the reproducing formula from Lemma 2 .

$$
\begin{aligned}
S_{t}\left(f_{x, k}\right)(z) & =\left(\frac{t}{\pi}\right)^{n} \int_{\mathbb{C}^{n}} e^{t\langle z, w\rangle} w_{1}^{k} e^{-(t+x)|w|^{2}} d v(w) \\
& =\left(\frac{t}{t+x}\right)^{n} \int_{\mathbb{C}^{n}} e^{(t+x)\langle t z /(t+x), w\rangle} w_{1}^{k} d v_{t+x}(w) \\
& =\left(\frac{t}{t+x}\right)^{n}\left(\frac{t z_{1}}{t+x}\right)^{k} \\
& =\left(\frac{t}{t+x}\right)^{n+k} z_{1}^{k} .
\end{aligned}
$$

We next calculate the norm of $S_{t}\left(f_{x, k}\right)$ in $L^{p}\left(\mathbb{C}^{n}, d v_{s}\right)$ with the help of Lemma1 again.

$$
\begin{aligned}
\int_{\mathbb{C}^{n}}\left|S_{t}\left(f_{x, k}\right)\right|^{p} d v_{s} & =\left(\frac{t}{t+x}\right)^{p(n+k)} \int_{\mathbb{C}^{n}}\left|z_{1}\right|^{p k} d v_{s}(z) \\
& =\left(\frac{t}{t+x}\right)^{p(n+k)} \frac{\Gamma((p k / 2)+1)}{s^{p k / 2}}
\end{aligned}
$$

Now if the integral operator $S_{t}$ is bounded on $L^{p}\left(\mathbb{C}^{n}, d v_{s}\right)$, then there exists a positive constant $C$ (independent of $x$ and $k$ ) such that

$$
\left(\frac{t}{t+x}\right)^{p(n+k)} \frac{\Gamma((p k / 2)+1)}{s^{p k / 2}} \leq C\left(\frac{s}{p x+s}\right)^{n} \frac{\Gamma((p k / 2)+1)}{(p x+s)^{p k / 2}},
$$

or

$$
\left(\frac{t}{t+x}\right)^{p(n+k)} \leq C\left(\frac{s}{s+p x}\right)^{n+(p k / 2)}
$$

Fix any $x>0$ and look at what happens in the above inequality when $k \rightarrow \infty$. We deduce that

$$
\left(\frac{t}{t+x}\right)^{2} \leq \frac{s}{s+p x}
$$

Cross multiply the two sides of the inequality above and simplify. The result is

$$
p t^{2} \leq 2 s t+s x .
$$

Let $x \rightarrow 0$. Then $p t^{2} \leq 2 s t$, or $p t \leq 2 s$. This completes the proof of the lemma.

Lemma 7. Suppose $1<p<\infty$ and $S_{t}$ is bounded on $L^{p}\left(\mathbb{C}^{n}, d v_{s}\right)$. Then $p t>s$. 
Proof. If $p>1$ and $S_{t}$ is bounded on $L^{p}\left(\mathbb{C}^{n}, d v_{s}\right)$, then $S_{t}^{*}$ is bounded on $L^{q}\left(\mathbb{C}^{n}, d v_{s}\right)$, where $1 / p+1 / q=1$. Applying the formula for $S_{t}^{*}$ from (4) to the constant function $f=1$ shows that the function $e^{(s-t)|z|^{2}}$ is in $L^{q}\left(\mathbb{C}^{n}, d v_{s}\right)$. From this we deduce that

$$
q(s-t)<s
$$

which is easily seen to be equivalent to $s<p t$.

Lemma 8. If $S_{t}$ is bounded on $L^{1}\left(\mathbb{C}^{n}, d v_{s}\right)$, then $t=2 s$.

Proof. Fix any $a \in \mathbb{C}^{n}$ and consider the function

$$
f_{a}(z)=\frac{e^{t\langle z, a\rangle}}{\left|e^{t\langle z, a\rangle}\right|}, \quad z \in \mathbb{C}^{n}
$$

Obviously, $\left\|f_{a}\right\|_{\infty}=1$ for every $a \in \mathbb{C}^{n}$. On the other hand, it follows from (4) and Lemma 3 that

$$
\begin{aligned}
S_{t}^{*}\left(f_{a}\right)(a) & =\left(\frac{t}{s}\right)^{n} e^{(s-t)|a|^{2}} \int_{\mathbb{C}^{n}}\left|e^{t\langle w, a\rangle}\right| d v_{s}(w) \\
& =\left(\frac{t}{s}\right)^{n} e^{(s-t)|a|^{2}} e^{t^{2}|a|^{2} /(4 s)}
\end{aligned}
$$

Since $S_{t}^{*}$ is bounded on $L^{\infty}\left(\mathbb{C}^{n}\right)$, there exists a positive constant $C$ such that

$$
\left(\frac{t}{s}\right)^{n} e^{(s-t)|a|^{2}} e^{t^{2}|a|^{2} /(4 s)} \leq\left\|S_{t}^{*}\left(f_{a}\right)\right\|_{\infty} \leq C\left\|f_{a}\right\|_{\infty}=C
$$

for all $a \in \mathbb{C}^{n}$. This clearly implies that

$$
s-t+\frac{t^{2}}{4 s} \leq 0
$$

which is equivalent to

$$
(2 s-t)^{2} \leq 0
$$

Therefore, we have $t=2 s$.

Lemma 9. Suppose $1<p \leq 2$ and $S_{t}$ is bounded on $L^{p}\left(\mathbb{C}^{n}, d v_{s}\right)$. Then $p t=2 s$.

Proof. Once again, we consider functions of the form

$$
f_{x, k}(z)=e^{-x|z|^{2}} z_{1}^{k}, \quad z \in \mathbb{C}^{n},
$$


where $x>0$ and $k$ is a positive integer. It follows from (4) and Lemma 2 that

$$
\begin{aligned}
S_{t}^{*}\left(f_{x, k}\right)(z) & =\left(\frac{t}{\pi}\right)^{n} e^{(s-t)|z|^{2}} \int_{\mathbb{C}^{n}} e^{t\langle z, w\rangle} w_{1}^{k} e^{-(s+x)|w|^{2}} d v(w) \\
& =\left(\frac{t}{s+x}\right)^{n} e^{(s-t)|z|^{2}} \int_{\mathbb{C}^{n}} e^{(s+x)\langle t z /(s+x), w\rangle} w_{1}^{k} d v_{s+x}(w) \\
& =\left(\frac{t}{s+x}\right)^{n} e^{(s-t)|z|^{2}}\left(\frac{t z_{1}}{s+x}\right)^{k} \\
& =\left(\frac{t}{s+x}\right)^{n+k} e^{(s-t)|z|^{2}} z_{1}^{k} .
\end{aligned}
$$

Suppose $1<p \leq 2$ and $1 / p+1 / q=1$. If the operator $S_{t}$ is bounded on $L^{p}\left(\mathbb{C}^{n}, d v_{s}\right)$, then the operator $S_{t}^{*}$ is bounded on $L^{q}\left(\mathbb{C}^{n}, d v_{s}\right)$. So there exists a positive constant $C$, independent of $x$ and $k$, such that

$$
\int_{\mathbb{C}^{n}}\left|S_{t}^{*}\left(f_{x, k}\right)\right|^{q} d v_{s} \leq C \int_{\mathbb{C}^{n}}\left|f_{x, k}\right|^{q} d v_{s} .
$$

It follows from the proof of Lemma 1 that

$$
\int_{\mathbb{C}^{n}}\left|f_{x, k}\right|^{q} d v_{s}=\left(\frac{s}{q x+s}\right)^{n} \frac{\Gamma((q k / 2)+1)}{(q x+s)^{q k / 2}} .
$$

On the other hand, it follows from Lemma 7 and its proof that $s-q(s-t)>$ 0 , so the integral

$$
I=\int_{\mathbb{C}^{n}}\left|S_{t}^{*}\left(f_{x, k}\right)\right|^{q} d v_{s}
$$

can be evaluated with the help of Lemma 1 as follows.

$$
\begin{aligned}
I & =\left(\frac{t}{s+x}\right)^{q(n+k)}\left(\frac{s}{\pi}\right)^{n} \int_{\mathbb{C}^{n}}\left|z_{1}\right|^{q k} e^{-(s-q(s-t))|z|^{2}} d v(z) \\
& =\left(\frac{t}{s+x}\right)^{q(n+k)}\left(\frac{s}{s-q(s-t)}\right)^{n} \int_{\mathbb{C}^{n}}\left|z_{1}\right|^{q k} d v_{s-q(s-t)}(z) \\
& =\left(\frac{t}{s+x}\right)^{q(n+k)}\left(\frac{s}{s-q(s-t)}\right)^{n} \frac{\Gamma((q k / 2)+1)}{(s-q(s-t))^{q k / 2}}
\end{aligned}
$$

Therefore,

$$
\left(\frac{t}{s+x}\right)^{q(n+k)}\left(\frac{s}{s-q(s-t)}\right)^{n} \frac{\Gamma((q k / 2)+1)}{(s-q(s-t))^{q k / 2}}
$$

is less than or equal to

$$
C\left(\frac{s}{q x+s}\right)^{n} \frac{\Gamma((q k / 2)+1)}{(q x+s)^{q k / 2}}
$$


which easily reduces to

$$
\left(\frac{t}{s+x}\right)^{q(n+k)} \leq C\left(\frac{s-q(s-t)}{s+q x}\right)^{n+(q k / 2)} .
$$

Once again, fix $x>0$ and let $k \rightarrow \infty$. We find out that

$$
\left(\frac{t}{s+x}\right)^{2} \leq \frac{s-q(s-t)}{s+q x}
$$

Using the relation $1 / p+1 / q=1$, we can change the right-hand side above to

$$
\frac{p t-s}{(p-1) s+p x}
$$

It follows that

$$
t^{2}(p-1) s+t^{2} p x \leq(p t-s)\left(s^{2}+2 s x+x^{2}\right)
$$

which can be written as

$$
(p t-s) x^{2}+\left[2 s(p t-s)-t^{2} p\right] x+s^{2}(p t-s)-t^{2}(p-1) s \geq 0 .
$$

Let $q(x)$ denote the quadratic function on the left-hand side of the above inequality. Since $p t-s>0$ by Lemma 7, the function $q(x)$ attains its minimum value at

$$
x_{0}=\frac{p t^{2}-2 s(p t-s)}{2(p t-s)} .
$$

Since $2 \geq p$, the numerator above is greater than or equal to

$$
p t^{2}-2 p t s+p s^{2}=p(t-s)^{2} .
$$

It follows that $x_{0} \geq 0$ and so $h(x) \geq h\left(x_{0}\right) \geq 0$ for all real $x$ (not just nonnegative $x$ ). From this we deduce that the discriminant of $h(x)$ cannot be positive. Therefore,

$$
\left[2 s(p t-s)-p t^{2}\right]^{2}-4(p t-s)\left[s^{2}(p t-s)-t^{2}(p-1) s\right] \leq 0 .
$$

Elementary calculations reveal that the above inequality is equivalent to

$$
(p t-2 s)^{2} \leq 0 \text {. }
$$

Therefore, $p t=2 s$.

Lemma 10. Suppose $2<p<\infty$ and $S_{t}$ is bounded on $L^{p}\left(\mathbb{C}^{n}, d v_{s}\right)$. Then $p t=2 s$.

Proof. If $S_{t}$ is bounded on $L^{p}\left(\mathbb{C}^{n}, d v_{s}\right)$, then $S_{t}^{*}$ is bounded on $L^{q}\left(\mathbb{C}^{n}, d v_{s}\right)$, where $1<q<2$ and $1 / p+1 / q=1$. It follows from (4) that there exists a positive constant $C$, independent of $f$, such that

$$
\int_{\mathbb{C}^{n}}\left|e^{(s-t)|z|^{2}} \int_{\mathbb{C}^{n}} e^{t\langle z, w\rangle}\left[f(w) e^{(t-s)|w|^{2}}\right] d v_{t}(w)\right|^{q} d v_{s}(z)
$$


is less than or equal to

$$
C \int_{\mathbb{C}^{n}}|f(w)|^{q} d v_{s}(w)
$$

where $f$ is any function in $L^{q}\left(\mathbb{C}^{n}, d v_{s}\right)$. Let

$$
f(z)=g(z) e^{(s-t)|z|^{2}},
$$

where $g \in L^{q}\left(\mathbb{C}^{n}, d v_{s-q(s-t)}\right)$ (recall from Lemma 7 that $s-q(s-t)>0$ ). We obtain another positive constant $\mathrm{C}$ (independent of $g$ ) such that

$$
\int_{\mathbb{C}^{n}}\left|S_{t} g\right|^{q} d v_{s-q(s-t)} \leq C \int_{\mathbb{C}^{n}}|g|^{q} d v_{s-q(s-t)}
$$

for all $g \in L^{q}\left(\mathbb{C}^{n}, d v_{s-q(s-t)}\right)$. Therefore, the operator $S_{t}$ is bounded on $L^{q}\left(\mathbb{C}^{n}, d v_{s-q(s-t)}\right)$. Since $1<q<2$, it follows from Lemma 9 that

$$
q t=2[s-q(s-t)] \text {. }
$$

It is easy to check that this is equivalent to $p t=2 s$.

We now complete the proof of the first part of the main theorem. As was pointed out in the introduction, this part of the theorem is known before. We included a full proof here for two purposes. First, this gives a different and self-contained approach. Second, as a by-product of this different approach, we are going to obtain the inequality $\left\|T_{t}\right\| \leq 2^{n}$, which is one half of the identity $\left\|T_{t}\right\|=2^{n}$.

Theorem 11. Suppose $t>0, s>0$, and $p \geq 1$. Then the following conditions are equivalent.

(a) The operator $T_{t}$ is bounded on $L^{p}\left(\mathbb{C}^{n}, d v_{s}\right)$.

(b) The operator $S_{t}$ is bounded on $L^{p}\left(\mathbb{C}^{n}, d v_{s}\right)$.

(c) The weight parameters satisfy $p t=2 s$.

Proof. When $p=1$, that (b) implies (c) follows from Lemma 8 , that (c) implies (a) follows from Fubini's theorem and Lemma 3, and that (a) implies (b) is obvious.

When $1<p<\infty$, that (b) implies (c) follows from Lemmas 9 and 10 , and that (a) implies (b) is still obvious.

So we assume $1<p<\infty$ and proceed to show that condition (c) implies (a). We do this with the help of Schur's test (Lemma 5).

Let $1 / p+1 / q=1$ and consider the positive function

$$
h(z)=e^{\lambda|z|^{2}}, \quad z \in \mathbb{C}^{n},
$$

where $\lambda$ is a constant to be specified later.

Recall that

$$
T_{t} f(z)=\int_{\mathbb{C}^{n}} H(z, w) f(w) d v_{s}(w),
$$


where

$$
H(z, w)=\left(\frac{t}{s}\right)^{n}\left|e^{t\langle z, w\rangle} e^{(s-t)|w|^{2}}\right|
$$

is a positive kernel. We first consider the integrals

$$
I(z)=\int_{\mathbb{C}^{n}} H(z, w) h(w)^{q} d v_{s}(w), \quad z \in \mathbb{C}^{n} .
$$

If $\lambda$ satisfies

$$
t>q \lambda
$$

then it follows from Lemma 3 that

$$
\begin{aligned}
I(z) & =\left(\frac{t}{\pi}\right)^{n} \int_{\mathbb{C}^{n}}\left|e^{t\langle z, w\rangle}\right| e^{-(t-q \lambda)|w|^{2}} d v(w) \\
& =\left(\frac{t}{t-q \lambda}\right)^{n} \int_{\mathbb{C}^{n}}\left|e^{t\langle z, w\rangle}\right| d v_{t-q \lambda}(w) \\
& =\left(\frac{t}{t-q \lambda}\right)^{n} e^{t^{2}|z|^{2} / 4(t-q \lambda)} .
\end{aligned}
$$

If we choose $\lambda$ so that

$$
\frac{t^{2}}{4(t-q \lambda)}=q \lambda
$$

then we obtain

$$
\int_{\mathbb{C}^{n}} H(z, w) h(w)^{q} d v_{s}(w) \leq\left(\frac{t}{t-q \lambda}\right)^{n} h(z)^{q}
$$

for all $z \in \mathbb{C}^{n}$.

We now consider the integrals

$$
J(w)=\int_{\mathbb{C}^{n}} H(z, w) h(z)^{p} d v_{s}(z), \quad w \in \mathbb{C}^{n} .
$$

If $\lambda$ satisfies

$$
s-p \lambda>0
$$

then it follows from Lemma 3 that

$$
\begin{aligned}
J(w) & =\left(\frac{t}{s}\right)^{n} \int_{\mathbb{C}^{n}}\left|e^{t\langle z, w\rangle} e^{(s-t)|w|^{2}}\right| h(z)^{p} d v_{s}(z) \\
& =\left(\frac{t}{\pi}\right)^{n} e^{(s-t)|w|^{2}} \int_{\mathbb{C}^{n}}\left|e^{t\langle z, w\rangle}\right| e^{-(s-p \lambda)|z|^{2}} d v(z) \\
& =\left(\frac{t}{s-p \lambda}\right)^{n} e^{(s-t)|w|^{2}} e^{t^{2}|w|^{2} / 4(s-p \lambda)} \\
& =\left(\frac{t}{s-p \lambda}\right)^{n} e^{\left[(s-t)+t^{2} / 4(s-p \lambda)\right]|w|^{2}}
\end{aligned}
$$


If we choose $\lambda$ so that

$$
s-t+\frac{t^{2}}{4(s-p \lambda)}=p \lambda
$$

then we obtain

$$
\int_{\mathbb{C}^{n}} H(z, w) h(z)^{p} d v_{s}(z) \leq\left(\frac{t}{s-p \lambda}\right)^{n} h(w)^{p}
$$

for all $w \in \mathbb{C}^{n}$. In view of Schur's test and the estimates in (8) and (11), we conclude that the operator $T_{t}$ would be bounded on $L^{p}\left(\mathbb{C}^{n}, d v_{s}\right)$ provided that we could choose a real $\lambda$ to satisfy conditions (6), (7), (9), and (10) simultaneously.

Under our assumption that $p t=2 s$ it is easy to verify that condition (7) is the same as condition (10). In fact, we can explicitly solve for $q \lambda$ and $p \lambda$ in (7) and (10), repectively, to obtain

$$
q \lambda=\frac{t}{2}, \quad p \lambda=\frac{2 s-t}{2} .
$$

The relations $p t=2 s$ and $1 / p+1 / q=1$ clearly imply that the two resulting $\lambda$ 's above are consistent, namely,

$$
\lambda=\frac{t}{2 q}=\frac{2 s-t}{2 p} .
$$

Also, it is easy to see that the above choice of $\lambda$ satisfies both (6) and (9). This completes the proof of the theorem.

Theorem 12. If $1 \leq p<\infty$ and $p t=2 s$, then

$$
\int_{\mathbb{C}^{n}}\left|S_{t} f\right|^{p} d v_{s} \leq \int_{\mathbb{C}^{n}}\left|T_{t} f\right|^{p} d v_{s} \leq 2^{n p} \int_{\mathbb{C}^{n}}|f|^{p} d v_{s}
$$

for all $f \in L^{p}\left(\mathbb{C}^{n}, d v_{s}\right)$.

Proof. With the choice of $\lambda$ in (12), the constants in (8) and (11) both reduce to $2^{n}$. Therefore, Schur's test tells us that, in the case when $1<p<\infty$, the norm of $T_{t}$ on $L^{p}\left(\mathbb{C}^{n}, d v_{s}\right)$ does not exceed $2^{n}$.

When $p=1$, the desired estimate follows from Fubini's theorem and Lemma 3 .

Theorem 12 above can be stated as $\left\|S_{t}\right\| \leq\left\|T_{t}\right\| \leq 2^{n}$, with $S_{t}$ and $T_{t}$ considered as operators on $L^{p}\left(\mathbb{C}^{n}, d v_{s}\right)$. We now proceed to the proof of the inequality $\left\|T_{t}\right\| \geq 2^{n}$. Several lemmas are needed for this estimate.

Lemma 13. For $c>0$ and $p \geq 1$ we have

$$
\lim _{h \rightarrow 0^{+}} h \int_{c}^{\infty}\left[\int_{c}^{\infty}(u v)^{-\frac{1}{4}} \exp \left(\sqrt{u v}-\frac{u+v}{2}-\frac{h v}{p}\right) d v\right]^{p} d u=(2 \sqrt{2 \pi})^{p} \text {. }
$$


Proof. We begin with the inner integral

$$
I(u)=\int_{c}^{\infty}(u v)^{-\frac{1}{4}} \exp \left(\sqrt{u v}-\frac{u+v}{2}-\frac{h v}{p}\right) d v .
$$

Let $a=\frac{1}{2}+\frac{h}{p}$ and change variables according to $v=t^{2}$. Then

$$
I(u)=2 u^{-\frac{1}{4}} e^{-\frac{u}{2}} \int_{\sqrt{c}}^{\infty} \sqrt{t} \exp \left(-a t^{2}+\sqrt{u} t\right) d t .
$$

Write

$$
-a t^{2}+\sqrt{u} t=-a\left(t-\frac{\sqrt{u}}{2 a}\right)^{2}+\frac{u}{4 a},
$$

make a change of variables according to $x=t-(\sqrt{u} / 2 a)$, and simplify the result. We obtain

$$
I(u)=2 u^{-\frac{1}{4}} e^{-\frac{u h}{2 a p}} \int_{\sqrt{c}-\frac{\sqrt{u}}{2 a}}^{\infty} \sqrt{x+\frac{\sqrt{u}}{2 a}} e^{-a x^{2}} d x .
$$

It is then clear that we can rewrite $I(u)$ as follows.

$$
I(u)=\varphi_{1}(u)+\varphi_{2}(u),
$$

where

$$
\varphi_{1}(u)=\frac{2}{\sqrt{2 a}} e^{-\frac{u h}{2 a p}} \int_{\sqrt{c}-\frac{\sqrt{u}}{2 a}}^{+\infty} e^{-a x^{2}} d x
$$

and

$$
\varphi_{2}(u)=\frac{2 e^{-\frac{u h}{2 a p}}}{u^{\frac{1}{4}}} \int_{\sqrt{c}-\frac{\sqrt{u}}{2 a}}^{+\infty} e^{-a x^{2}}\left(\sqrt{x+\frac{\sqrt{u}}{2 a}}-\sqrt{\frac{\sqrt{u}}{2 a}}\right) d x .
$$

For the function $\varphi_{2}$ we rationalize the numerator in its integrand to obtain

$$
\begin{aligned}
\left|\varphi_{2}(u)\right| & \leq \frac{2}{u^{\frac{1}{4}}} e^{-\frac{u h}{2 a p}} \int_{\sqrt{c}-\frac{\sqrt{u}}{2 a}}^{+\infty} e^{-a x^{2}} \frac{|x|}{\sqrt{\frac{\sqrt{u}}{2 a}}} d x \\
& \leq \frac{2 \sqrt{2 a}}{u^{\frac{1}{2}}} e^{-\frac{u h}{2 a p}} \int_{-\infty}^{+\infty}|x| e^{-a x^{2}} d x .
\end{aligned}
$$

A simple calculation of the last integral above then gives

$$
\left|\varphi_{2}(u)\right| \leq \frac{4}{\sqrt{2 a}} u^{-\frac{1}{2}} e^{-\frac{u h}{2 a p}}
$$

Similarly, we have

$$
\left|\varphi_{1}(u)\right| \leq \frac{2}{\sqrt{2 a}} e^{-\frac{u h}{2 a p}} \int_{-\infty}^{\infty} e^{-a x^{2}} d x=\frac{\sqrt{2 \pi}}{a} e^{-\frac{u h}{2 a p}}
$$


We now use the above estimates to show that

$$
\lim _{h \rightarrow 0^{+}} h \int_{c}^{\infty} \varphi_{1}(u)^{p-1}\left|\varphi_{2}(u)\right| d u=0
$$

and

$$
\lim _{h \rightarrow 0^{+}} h \int_{c}^{\infty}\left|\varphi_{2}(u)\right|^{p} d u=0 .
$$

In fact, according to (13) and (14),

$$
h \varphi_{1}(u)^{p-1}\left|\varphi_{2}(u)\right| \leq\left(\frac{\sqrt{2 \pi}}{a}\right)^{p-1} \frac{4}{\sqrt{2 a}} h u^{-\frac{1}{2}} e^{-\frac{u h}{2 a}},
$$

from which we derive that

$$
\begin{aligned}
h \int_{c}^{\infty} \varphi_{1}(u)^{p-1}\left|\varphi_{2}(u)\right| d u & \leq\left(\frac{\sqrt{2 \pi}}{a}\right)^{p-1} h \frac{4}{\sqrt{2 a}} \int_{c}^{\infty} u^{-\frac{1}{2}} e^{-\frac{u h}{2 a}} d u \\
& =4\left(\frac{\sqrt{2 \pi}}{a}\right)^{p-1} \sqrt{h} \int_{\frac{c h}{2 a}}^{+\infty} \omega^{-\frac{1}{2}} e^{-\omega} d \omega
\end{aligned}
$$

Since $a \rightarrow \frac{1}{2}$ as $h \rightarrow 0^{+}$, we obtain (15). On the other hand, it follows from (13) that

so

$$
\left|\varphi_{2}(u)\right|^{p} \leq\left(\frac{4}{\sqrt{2 a}}\right)^{p} u^{-\frac{p}{2}} e^{-\frac{u h}{2 a}}
$$

$$
\begin{aligned}
h \int_{c}^{\infty}\left|\varphi_{2}(u)\right|^{p} d u & \leq\left(\frac{4}{\sqrt{2 a}}\right)^{p} h \int_{c}^{\infty} u^{-\frac{p}{2}} e^{-\frac{u h}{2 a}} d u= \\
& =2^{p-1} a^{1-p} h^{p / 2} \int_{\frac{c h}{2 a}}^{+\infty} \omega^{-\frac{p}{2}} e^{-\omega} d \omega .
\end{aligned}
$$

Let $h \rightarrow 0^{+}$and use the fact that $a \rightarrow \frac{1}{2}$ as $h \rightarrow 0^{+}$. We obtain (16).

By the change of variables $s=u h / 2 a$, we have

$$
\begin{aligned}
h \int_{c}^{\infty} \varphi_{1}(u)^{p} d u & =h \int_{c}^{\infty}\left(\frac{2}{\sqrt{2 a}}\right)^{p} e^{-\frac{u h}{2 a}}\left(\int_{\sqrt{c}-\frac{\sqrt{u}}{2 a}}^{+\infty} e^{-a x^{2}} d x\right)^{p} d u \\
& =\frac{2^{p+1} a}{(\sqrt{2 a})^{p}} \int_{\frac{h c}{2 a}}^{\infty} e^{-s}\left(\int_{\sqrt{c}-\frac{\sqrt{s}}{\sqrt{2 a h}}}^{+\infty} e^{-a x^{2}} d x\right)^{p} d s
\end{aligned}
$$

Let $h \rightarrow 0^{+}$, notice that $a \rightarrow \frac{1}{2}$ as $h \rightarrow 0^{+}$, and use Lebesgue's dominated convergence theorem. We get

$$
\lim _{h \rightarrow 0^{+}} h \int_{c}^{\infty} \varphi_{1}^{p} d u=2^{p} \int_{0}^{\infty} e^{-s}\left[\int_{-\infty}^{+\infty} e^{-\frac{x^{2}}{2}} d x\right]^{p} d s=(2 \sqrt{2 \pi})^{p} .
$$


If $p \geq 1$, it is easy to see that the function

$$
g(z)=\frac{|1+z|^{p}-|z|^{p}}{1+|z|^{p-1}}
$$

is continuous and bounded on $\mathbb{C}$. Replacing $z$ by $z / w$, we see that

$$
|| z+\left.w\right|^{p}-|z|^{p} \mid \leq C\left(|z|^{p-1}|w|+|w|^{p}\right)
$$

for all $z$ and $w$, where $C$ is a positive constant that only depends on $p$. This along with (15) and (16) shows that

$$
\lim _{h \rightarrow 0^{+}} h \int_{c}^{\infty}\left|\left(\varphi_{1}(u)+\varphi_{2}(u)\right)^{p}-\varphi_{1}(u)^{p}\right| d u=0 .
$$

Combining this with (17), we conclude that

$$
\lim _{h \rightarrow 0^{+}} h \int_{c}^{\infty}\left(\varphi_{1}(u)+\varphi_{2}(u)\right)^{p} d u=(2 \sqrt{2 \pi})^{p} .
$$

This proves the desired result.

Lemma 14. Let

$$
\mathcal{K}(x, y)=\sum_{n=0}^{\infty} \frac{t^{2 n} x^{n} y^{n}}{4^{n}(n !)^{2}}
$$

and define an integral operator

$$
A: L^{p}(0, \infty) \rightarrow L^{p}(0, \infty)
$$

by

$$
A f(x)=\int_{0}^{\infty} t e^{-\frac{t}{2}(x+y)} \mathcal{K}(x, y) f(y) d y
$$

where $p \geq 1$ and $t$ is any fixed positive constant. Then the norm of $A$ on $L^{p}(0, \infty)$ satisfies $\|A\| \geq 2$.

Proof. It follows from the asymptotic behavior of the Bessel function $J_{0}(x)$ (see page 199 of [17] for example) that

$$
\sum_{k=0}^{\infty} \frac{(z / 2)^{2 k}}{(k !)^{2}} \sim \frac{e^{z}}{\sqrt{2 \pi z}}
$$

as $z \rightarrow \infty$. Thus

$$
\sum_{n=0}^{\infty} \frac{u^{n}}{(n !)^{2}} \sim \frac{e^{2 \sqrt{u}}}{\sqrt{4 \pi} u^{\frac{1}{4}}}
$$

as $u \rightarrow \infty$. Fix an arbitrary $\eta>0$ and choose some $u_{0}>0$ such that

$$
\sum_{n=0}^{\infty} \frac{u^{n}}{(n !)^{2}}>(1-\eta) \frac{e^{2 \sqrt{u}}}{\sqrt{4 \pi} u^{\frac{1}{4}}}
$$

for every $u \geq u_{0}$. 
Let $\delta_{0}=2 \sqrt{u_{0}} / t$. It follows from (18) that

$$
\mathcal{K}(x, y)>(1-\eta) \frac{e^{t \sqrt{x y}}}{(x y)^{\frac{1}{4}} \sqrt{2 \pi t}}
$$

for all $x \geq \delta_{0}$ and all $y \geq \delta_{0}$. Fix a positive number $\varepsilon$ and let

$$
f_{\varepsilon}(x)=\exp \left(-\frac{\varepsilon x}{p}\right) \text {. }
$$

Then

and so

$$
\left\|f_{\varepsilon}\right\|=\left[\int_{0}^{\infty}\left|f_{\varepsilon}(x)\right|^{p} d x\right]^{1 / p}=\varepsilon^{-\frac{1}{p}}
$$

$$
\|A\| \geq \varepsilon^{\frac{1}{p}}\left\|A f_{\varepsilon}\right\| .
$$

On the other hand, it follows from (19) that

$$
\begin{aligned}
\left\|A f_{\varepsilon}\right\| & \geq\left(\int_{\delta_{0}}^{\infty}\left|A f_{\varepsilon}(x)\right|^{p} d x\right)^{\frac{1}{p}} \\
& \geq\left[\int_{\delta_{0}}^{\infty} d x\left(\int_{\delta_{0}}^{\infty} t e^{-\frac{t}{2}(x+y)} \mathcal{K}(x, y) e^{-\frac{\varepsilon y}{p}} d y\right)^{p}\right]^{\frac{1}{p}} \\
& \geq \frac{(1-\eta) t}{\sqrt{2 \pi t}}\left[\int_{\delta_{0}}^{\infty} d x\left(\int_{\delta_{0}}^{\infty} \frac{e^{-\frac{t}{2}(x+y)+t \sqrt{x y}-\frac{\varepsilon y}{p}}}{(x y)^{\frac{1}{4}}} d y\right)^{p}\right]^{\frac{1}{p}} .
\end{aligned}
$$

Combining this with (20), we obtain

$$
\|A\| \geq \frac{(1-\eta) t}{\sqrt{2 \pi t}}\left[\varepsilon \int_{\delta_{0}}^{\infty} d x\left(\int_{\delta_{0}}^{\infty} \frac{e^{-\frac{t}{2}(x+y)+t \sqrt{x y}-\frac{\varepsilon y}{p}}}{(x y)^{\frac{1}{4}}} d y\right)^{p}\right]^{\frac{1}{p}} .
$$

After the change of variables $x t=u$ and $y t=v$ we obtain

$$
\|A\| \geq \frac{1-\eta}{\sqrt{2 \pi}}\left[\frac{\varepsilon}{t} \int_{t \delta_{0}}^{\infty} d u\left(\int_{t \delta_{0}}^{\infty} \frac{e^{-\frac{1}{2}(u+v)+\sqrt{u v}-\frac{\varepsilon v}{t p}}}{(u v)^{\frac{1}{4}}} d v\right)^{p}\right]^{\frac{1}{p}} .
$$

Let $t \delta_{0}=c$ and $\varepsilon / t=h$. Clearly, $h \rightarrow 0^{+}$when $\varepsilon \rightarrow 0^{+}$. Let $\varepsilon \rightarrow 0^{+}$in (21) and apply Lemma 13 . We obtain

$$
\|A\| \geq \frac{1-\eta}{\sqrt{2 \pi}} \cdot 2 \sqrt{2 \pi}=2(1-\eta) .
$$

Since $\eta>0$ is arbitrary, we obtain $\|A\| \geq 2$, and the proof of Lemma 14 is complete.

We can now prove the main result of the paper. 
Theorem 15. If $1 \leq p<\infty$ and $p t=2 s$, then the norm of $T_{t}$ on $L^{p}\left(\mathbb{C}^{n}, d v_{s}\right)$ is given by $\left\|T_{t}\right\|=2^{n}$.

Proof. In view of Theorem 12 it is enough for us to prove the inequality $\left\|T_{t}\right\| \geq 2^{n}$.

Recall that when $n=1$, we use the notation $d A_{s}$ instead of $d v_{s}$. For $f \in L^{p}\left(\mathbb{C}, d A_{s}\right)$ we consider

$$
\Phi\left(z_{1}, \cdots, z_{n}\right)=f\left(z_{1}\right) \cdots f\left(z_{n}\right)
$$

Then $\Phi \in L^{p}\left(\mathbb{C}^{n}, d v_{s}\right)$ and we have

$$
\left\|T_{t}\right\|^{p} \geq \frac{\left\|T_{t} \Phi\right\|^{p}}{\|\Phi\|^{p}}=\left[\frac{\int_{\mathbb{C}}\left|\int_{\mathbb{C}}\right| e^{t z \bar{\zeta}}\left|f(\zeta) d A_{t}(\zeta)\right|^{p} d A_{s}(z)}{\int_{\mathbb{C}}|f(\zeta)|^{p} d A_{s}(\zeta)}\right]^{n} .
$$

When $f$ runs over all unit vectors in $L^{p}\left(\mathbb{C}, d A_{s}\right)$, the supremum of the quotient inside the brackets above is exactly the $p$ th power of the norm of the operator $T_{t}$ on $L^{p}\left(\mathbb{C}, d A_{s}\right)$. So we only need to prove the inequality $\left\|T_{t}\right\| \geq 2^{n}$ for $n=1$.

Now we assume $n=1, p \geq 1$, and let

$$
T_{t}: L^{p}\left(\mathbb{C}, d A_{s}\right) \rightarrow L^{p}\left(\mathbb{C}, d A_{s}\right)
$$

be the integral operator defined by

$$
T_{t} f(z)=\int_{\mathbb{C}}\left|e^{t z \bar{\zeta}}\right| f(\zeta) d A_{t}(\zeta)
$$

To obtain a lower estimate of the norm of $T_{t}$ on $L^{p}\left(\mathbb{C}, d A_{s}\right)$, we apply $T_{t}$ to a family of special functions. More specifically, we consider functions of the form

$$
f(z)=G\left(|z|^{2}\right) e^{t|z|^{2} / 2}, \quad z \in \mathbb{C}
$$

where $G$ is any unit vector in $L^{p}(0, \infty)$. It follows from polar coordinates and the assumption $p t=2 s$ that

$$
\|f\|^{p}=\int_{\mathbb{C}}|f|^{p} d A_{s}=s \int_{0}^{\infty}|G(x)|^{p} d x=s .
$$


On the other hand,

$$
\begin{aligned}
T_{t} f(z) & =\int_{\mathbb{C}}\left|e^{t z \bar{w}}\right| G\left(|w|^{2}\right) e^{t|w|^{2} / 2} d A_{t}(w) \\
& =\int_{\mathbb{C}}\left|e^{t z \bar{w} / 2}\right|^{2} G\left(|w|^{2}\right) e^{t|w|^{2} / 2} d A_{t}(w) \\
& =\int_{\mathbb{C}}\left|\sum_{n=0}^{\infty} \frac{(t z \bar{w} / 2)^{n}}{n !}\right|^{2} G\left(|w|^{2}\right) e^{t|w|^{2} / 2} d A_{t}(w) \\
& =\sum_{n=0}^{\infty} \frac{t^{2 n}|z|^{2 n}}{4^{n}(n !)^{2}} \int_{\mathbb{C}}|w|^{2 n} G\left(|w|^{2}\right) e^{t|w|^{2} / 2} d A_{t}(w) \\
& =\sum_{n=0}^{\infty} \frac{t^{2 n}|z|^{2 n}}{4^{n}(n !)^{2}} t \int_{0}^{\infty} y^{n} G(y) e^{-t y / 2} d y \\
& =\int_{0}^{\infty} t e^{-t y / 2} \mathcal{K}\left(|z|^{2}, y\right) G(y) d y \\
& =e^{t|z|^{2} / 2} A G\left(|z|^{2}\right),
\end{aligned}
$$

where the kernel $\mathcal{K}$ and the operator $A$ are from Lemma 14 Using polar coordinates and the assumption $p t=2 s$ one more time, we obtain

$$
\left\|T_{t} f\right\|^{p}=\int_{\mathbb{C}}\left|T_{t} f(z)\right|^{p} d A_{s}(z)=s \int_{0}^{\infty}|A G(x)|^{p} d x .
$$

By (22) and (23) we must have

$$
\left\|T_{t}\right\|^{p} \geq \frac{\left\|T_{t} f\right\|^{p}}{\|f\|^{p}}=\int_{0}^{\infty}|A G(x)|^{p} d x .
$$

Take the supremum over $G$ and apply Lemma 14 , The result is

$$
\left\|T_{t}\right\|^{p} \geq\|A\|^{p} \geq 2^{p} .
$$

This completes the proof of the theorem.

We conclude the paper with two corollaries.

Corollary 16. For any $s>0$ and $p \geq 1$ the Fock space $F_{s}^{p}$ is a complemented subspace of $L^{p}\left(\mathbb{C}^{n}, d v_{s}\right)$, that is, there exists a closed subspace $X_{s}^{p}$ of $L^{p}\left(\mathbb{C}^{n}, d v_{s}\right)$ such that

$$
L^{p}\left(\mathbb{C}^{n}, d v_{s}\right)=F_{s}^{p} \oplus X_{s}^{p}
$$

where $\oplus$ denotes the direct sum of two subspaces.

Proof. Choose $t>0$ such that $p t=2 s$. Then by Theorem 11, the operator $S_{t}$ is a bounded projection from $L^{p}\left(\mathbb{C}^{n}, d v_{s}\right)$ onto $F_{s}^{p}$. This shows that $F_{s}^{p}$ is complemented in $L^{p}\left(\mathbb{C}^{n}, d v_{s}\right)$. 
The following result is obviously a generalization of Theorem 11, but it is also a direct consequence of Theorem 11.

Corollary 17. Suppose $a>0, b>0, s>0$, and $p \geq 1$. Then the following conditions are equivalent.

(a) The integral operator

$$
T_{a, b} f(z)=\int_{\mathbb{C}^{n}}\left|e^{-a|z|^{2}+(a+b)\langle z, w\rangle-b|w|^{2}}\right| f(w) d v(w)
$$

is bounded on $L^{p}\left(\mathbb{C}^{n}, d v_{s}\right)$.

(b) The integral operator

$$
S_{a, b} f(z)=\int_{\mathbb{C}^{n}} e^{-a|z|^{2}+(a+b)\langle z, w\rangle-b|w|^{2}} f(w) d v(w)
$$

is bounded on $L^{p}\left(\mathbb{C}^{n}, d v_{s}\right)$.

(c) The parameters satisfy $p(a+b)=2(s+p a)$.

Proof. The boundedness of $S_{a, b}$ on $L^{p}\left(\mathbb{C}^{n}, d v_{s}\right)$ is equivalent to the existence of a positive constant $C$, independent of $f$, such that

$$
\int_{\mathbb{C}^{n}}\left|\int_{\mathbb{C}^{n}} e^{(a+b)\langle z, w\rangle-b|w|^{2}} f(w) d v(w)\right|^{p} d v_{s+p a}(z)
$$

is less than or equal to

$$
C \int_{\mathbb{C}^{n}}\left|f(z) e^{a|z|^{2}}\right|^{p} d v_{s+p a}(z) .
$$

Replacing $f(z)$ by $f(z) e^{-a|z|^{2}}$, we see that the above condition is equivalent to

$$
\int_{\mathbb{C}^{n}}\left|\int_{\mathbb{C}^{n}} e^{(a+b)\langle z, w\rangle} f(w) d v_{a+b}(w)\right|^{p} d v_{s+p a}(z) \leq C \int_{\mathbb{C}^{n}}|f|^{p} d v_{s+p a} .
$$

This is clearly equivalent to the boundedness of $S_{a+b}$ on $L^{p}\left(\mathbb{C}^{n}, d v_{s+p a}\right)$, which, according to Theorem 11, is equivalent to $p(a+b)=2(s+p a)$. Therefore, conditions (b) and (c) are equivalent. The equivalence of (a) and (c) is proved in exactly the same way.

\section{REFERENCES}

[1] V. Bargmann, On a Hilbert space of analytic functions and an associated integral transform, Comm. Pure. Appl. Math. 14 (1961), 187-214.

[2] C. Berger and L. Coburn, Toeplitz operators on the Segal-Bargmann space, Trans. Amer. Math. Soc. 301 (1987), 813-829.

[3] C. Berger and L. Coburn, Heat flow and Berezin-Toeplitz estimates, Amer. J. Math. 116 (1994), 563-590.

[4] C. Berger, L. Coburn, and K. Zhu, Toeplitz operators and function theory in ndimensions, Springer Lecture Notes in Mathematics 1256 (1987), 28-35. 
[5] M. Dostanić, Norm estimate of the Cauchy transform on $L^{p}(\Omega)$, Integral Equations and Operator Theory 52 (2005), 465-475.

[6] M. Dostanić, Norm of the Berezin transform on $L^{p}$ spaces, to appear in J. d'Analyse Math.

[7] G. Folland, Harmonic Analysis in Phase Space, Ann. Math. Studies 122, Princeton University Press, 1989.

[8] F. Forelli and W. Rudin, Projections on spaces of holomorphic functions in balls, Indiana Univ. Math. J. 24 (1974), 593-602.

[9] V. Guillemin, Toeplitz operators in $n$ dimensions, Integral Equations and Operator Theory 7 (1984), 145-205.

[10] P. Halmos and V. Sunder, Bounded Integral Operators on $L^{2}$ Spaces, Springer-Verlag, Berlin, 1978.

[11] S. Janson, J. Peetre, and R. Rochberg, Hankel forms and the Fock space, Revista Mat. Ibero-Amer. 3 (1987), 61-138.

[12] O. Kures and K. Zhu, A class of integral operators on the unit ball of $\mathbb{C}^{n}$, to appear in Integral Equations and Operator Theory.

[13] S.K. Pichorides, On the best values of the constants in the theorems of M. Riesz, Zygmund, and Kolmogorov, Studia Math. 44 (1972), 165-179.

[14] W. Rudin, Function Theory in the Unit Ball of $\mathbb{C}^{n}$, Springer-Verlag, New York, 1980.

[15] P. Sjögren, Un contre-exemple pour le noyau reproduisant de la mesure gaussienne dans le plan complexe, Seminaire Paul Krée (Equations aux dérivées partielles en dimension infinite), Paris, 1975/1976.

[16] Y.-C. Tung, Fock spaces, Ph.D. dissertation at the University of Michigan, 2005.

[17] G.N. Watson, A Treatise of the Theory of Bessel Functions, second edition, Cambridge University Press, 1995.

[18] J. Xia and D. Zheng, Standard deviation and Schatten class Hankel operators on the Segal-Bargmann space, Indiana Univ. Math. J. 53 (2004), 1381-1399.

[19] K. Zhu, A Forelli-Rudin type theorem, Complex Variables 16 (1991), 107-113.

[20] K. Zhu, Spaces of Holomorphic Functions in the Unit Ball, Springer-Verlag, New York, 2004.

[21] K. Zhu, A sharp norm estimate of the Bergman projection on $L^{p}$ spaces, Contemporary Math. 404 (2006), 199-205.

MatematičKi fakultet, Studentski trg 16, 11000 Beograd, Serbia

E-mail address: domi@matf.bg.ac.yu

DEPARTMENT OF MATHEMATICS, SUNY, Albany, NY 12222, USA

E-mail address: kzhu@math. albany.edu 